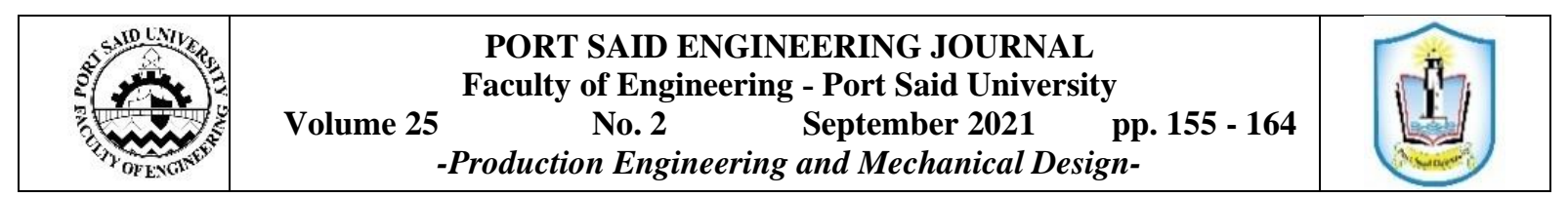

\title{
A Novel Approach for Optimizing Real Life Problems Using Hybrid Genetic Algorithm and Inner - Outer Array
}

\author{
Omnia O. Fadel ${ }^{1, a}$, Mohamed H. Gadallah ${ }^{2}$, Hanan K. Kouta ${ }^{1}$, Mohamed A. Zaghloul ${ }^{1}$
}

\begin{abstract}
One issue with optimization is that when the problem becomes more complicated, the stand-alone optimizer is unable to obtain the global optimal value consistently. That is why the Inner-Outer Array is developed to help the optimizer find a global optimum without going too deeply into the optimizer's parameter settings, which are not always applicable. As a result, this paper presents a novel hybridization approach combining Inner-Outer Array (IOA) and Genetic Algorithm (GA). IOA is a critical step in the IOA-GA method since it aids in the discovery of the global or near-global optimal solution. The developed approach, known as the Inner-Outer Array (IOA), is based on two stages of experimental design: parameter design and tolerance design. Depending on the number of variables and constraints vs. problem size, this approach has one inner array and one or more outer arrays. During the preceding few decades, genetic algorithms (GAs) have proven to be an effective technique for solving real-world optimization problems. In the case of a wide solution space and multiple local optima, however, GAs cannot guarantee a global optimum solution. And here comes the role of the exploratory ability of the inner-outer array (IOA) in scoping the search space, including guiding the genetic algorithm (GA) to reach the global or near global optimal result, which is the purpose of this work. More than 15 complex engineering optimization applications, inspired by real problems in the field of mechanical engineering, are used to verify the performance of the proposed method IOA-GA. This research paper used two issues from the literature: Himmelblau's non-linear optimization issue and Pressure Vessel Design. The results are then compared to other, complex and well-known algorithms. When compared to existing hybridization procedures, the results show that the suggested method is capable. Finally, the IOA-GA method is comparable to other effective methods.
\end{abstract}

Keywords: Hybrid genetic algorithm, optimization, Inner-Outer Array, Initial population, Engineering design problems

\section{INTRODUCTION}

Every day, many optimization problems are encountered, which are found in many scientific disciplines around us, such as engineering, manufacturing, aerospace, economic, medicine and more. Therefore, in recent years, optimization has attracted a lot of attention, and many new optimization approaches and techniques have been developed and applied to real-world issues. The aim of developing them is to find out global or near global optimum solution using a huge search space like genetic algorithm GAand simulated annealing SA.

${ }^{1}$ Production Engineering \& Mechanical Design Department, Faculty of Engineering, Port Said University

a email: omniaoyman@eng.psu.edu.eg, Corresponding author

${ }^{2}$ Production Engineering \& Mechanical Design Department, Faculty of Engineering, Cairo University, Giza, Egypt, email: mohamed@aucegypt.edu

Received: 17-06-2021, Accepted: 08-08-2021 DOI: 10.21608/PSERJ.2021.81050.1122
In this section, some of these hybrid algorithms, which presented by different researchers, are summarized. The Inner-Outer algorithm is presented as a competitive Accordingly, a lot of researchers subtracted numerous combinations of local search approaches with different optimization algorithms to take their benefits and reduce their flaws in discovering the optimal solution [1]. algorithm with other complex hybridization optimization algorithms by Fadel et al. [2].

It was developed to solve constrained optimization problems based on principles of experimental design and tolerance design approaches. The outcome indicated that InOut algorithm was an effective and efficient tool to solve constrained optimization problems with needs to little improvements. To handle global optimization problems, Tasi et al. [3] suggested a novel methodology based on merging the Taguchi method and a standard genetic algorithm. The goal of this hybridization was to take use of GA's great ability to explore global optima and Taguchi's ability to screen the best chromosomes. In addition to the technique described by Vaghela and Prajapati [4], when a 
hybrid strategy of Taguchi and genetic algorithm was proposed to reduce the number of repetitions using the powerful of OA to determine the initial population for GA, the proposed approach was used to 15 benchmark issues in order to study the performance of hybrid algorithm and then compare the findings. Karen et al. [5] proposed a hybrid approach based on the genetic algorithm and Taguchi method to solve a real-world problem in the design and manufacturing field. The hybrid algorithm was applied to vehicle component. The problems related to automotive industry are usually multi-objective problems. The proposed approach has been subjected to verification by evaluating both of fatigue damage and life of two-bar truss. It was found by results that the hybrid algorithm is more effective than classical optimization methods. Stjepan et al. [6] investigated the effects of eight different types of crossover operators on the performance of the hybrid Taguchi-genetic algorithm. The investigation's goal was to show how to use the method to continuous optimization. The various crossover operators were put to the test using 15 different test functions as case studies for each operator. In addition to roulette wheel selection, it was discovered that the optimal crossover operator for successful hybrid Taguchi genetic algorithm performance was segmented.

Bernardino et al. [7] proposed a hybridization approach depends on genetic algorithm (GA) and artificial immune system (AIS). The proposed algorithm was applied to a set of constrained optimization problems related to mechanical engineering. The hybrid algorithm AIS-GA has been verified by comparing its findings with the result of alternative approaches. The comparative results showed the effectiveness of the proposed approach in the problems that contain continuous design variables. However, it is less effective in problems that contain discrete design variables. A hybrid genetic algorithm was proposed by Chan et al. [8] as an approach for optimizing problems relate to production and distribution in supply chain area. The problem contained multi-factory model which required to make a critical decision because it depends on several criterions such as resources utilization, cost of operating and service level. It was found that the proposed optimization approach is very beneficial for obtaining reliable and robust solutions related to supply chain models. Mala et al. [9] seek to improve the quality of test cases by minimizing the error as possible as they can. They aimed to achieve results near global optima so that they proposed a hybrid approach based on genetic algorithm (HGA). The proposed hybrid algorithm, simple genetic algorithm (SGA) and Bacteriologic algorithm (BA) were applied to test cases and then compared the results. Result was concluded that HGA was more effective and efficient than SGA and BA. A hybridization approach combining Artificial Neural Network (ANN) and Genetic Algorithm (GA) was presented in several works. The hybrid ANN-GA $[10,11]$ was developed to optimize parameters of machining that contribute in minimizing surface roughness. It was found that the optimal value of parameters could be predicted by using the hybrid ANN-GA. A hybrid Particle Swarm
Optimization and genetic Algorithm (HPSOGA) has been proposed by Ali and Tawhid [12], in order to optimize a simplified model of the molecule's energy function. A set of benchmark problems has been studied using that hybrid algorithm in respect of investigating its performance on large scale problems. Computational results showed that the proposed algorithm able to reach the optimal or near to global optimal rapidly. Javaid et al. [13] combined traditional Genetic (GA) with a Local Search Heuristic (LSH) to fix problems related to cell formation. A wide range of problems has been solved by hybrid genetic algorithm (HGA) and GA. The evaluation and convergence abilities of HGA and GA have been compared. HGA proved its efficiency and accuracy especially with increasing scale of problems unlike traditional GA. Al-Milli [14] developed an approach coupling Genetic Algorithm and Deluge Algorithm. That hybridization exploits the optimum exploration of GA and gaudiness of deluge algorithm. Improving the exploration speed of GA was the objective of that study. The hybrid approach has been used to solve a set of nonlinear problems.

The performance of the proposed approach on solving mechanical engineering problems was satisfying. One of the newest approaches are proposed under that topic, in the research of Zhange et al. [15] a novel proposal approach integrates a genetic algorithm (GA), an artificial neural network (ANN), multivariate regression analysis (MRA), and a fuzzy logic controller (FLC) to improve the inside environment and energy consumption of building design.

Additionally, a hybrid technique was developed by Yassin et al. [16] depends on genetic algorithm, the sequential quadratic programming and on the particle swarm optimization called GA-PSO-SQP. This procedure was suggested for resolution constrained engineering optimization problems. Then compared their result with others heuristic procedures to prove the capability of proposed algorithm. Moreover, Duary et al. [17] proposed a combination of real-coded genetic algorithm and selforganizing migrating algorithm (RCSOMGA) to evaluate the global or near global optimum of nonlinear constrained optimization problems.

Based on what has been concluded from previous research, the proposed method is presented to enhance performance of GA in solving constrained and unconstrained optimization problems by overcoming the local optima and reaching directly to the global optimality. This proposed approach utilizes the power of Inner-Outer array (IOA) in exploitation optimum individuals and then providing them as an initial population matrix to GA.

\section{METHODS}

This research used a unique approach to narrow down the fitness function's search space. The suggested approach is based on the foundations of orthogonal arrays and experimental design, which are used to sample information about the fitness function and identify which independent factors have a substantial impact on the fitness function. The proposed algorithm will be demonstrated in this section 
before being applied to complicated real-world engineering design challenges in the following section. The fundamentals of genetic algorithm GA will be briefly introduced before the suggested algorithm IOA-GA is presented.

\subsection{Genetic Algorithm (GA)}

There are several approaches for optimizing a solution. One of them is the genetic algorithm, which is used for finding global optimality. GA depends on well-known steps and operations like selection, crossover and mutation, as shown in Figure 1, each one of them influences its performance in choosing the correct path to get the global optimal result. Initial Population is one of these steps, which is chosen by a random search. It plays an important role in determining the correct path of the GA, and also affects the number of iterations.

\subsection{Inner-Outer Algorithm (IOA)}

Inner-Outer Algorithm (IOA) is developed by Fadel et al. [2], the main idea of it based on design of experiments basics, where two key phases are required to be passed through in order to obtain the optimal value of the objective function. First phase is designing inner array for less sensitive factors and outer array for affected factors on system. The second phase is designing tolerance for the best condition which was resulted from the first phase. This algorithm has been proposed to solve optimization problems and then be a competitor for stochastic optimization algorithms and their hybridizations like Genetic Algorithm (GA), Simulated Annealing (SA), Particle Swarm Optimization (PSO), etc. It can also be combined with any optimizer to improve its performance and overcome its defects, and in turn, it can guide optimization techniques to reach global optima, the researchers' aim.

\subsection{Hybrid Inner-Outer Algorithm and Genetic Algorithm (IOA-GA)}

The majority of hybridization, particularly utilizing the Taguchi technique, has concentrated on selecting the initial population. Rather than using a random search to select initial population, in this research, inner- outer arrays are used to pick the initial population of GA which helps in reducing iterations number. The main idea of proposed IOA-GA is inserting the best results of inner-outer algorithm and tolerance design stage as an initial population for GA to start its generation from it as shown in next proposed model Figure 2. The proposed hybrid GA utilized Inner-Outer algorithm in searching for a set of the best solutions, as local optimum solutions, that will reduce number of iterations of GA.

\section{MATHEMATICAL MODELS OF CONSTRAINED ENGINEERING OPTIMIZATION PROBLEMS}

In the real world, most engineering design problems are complicated, as they are non-linear or large-scale, and they must be handled under constraints. To verify the validity of the proposed algorithm, fifteen engineering design optimization issues were used, although only two are described here. All of these problems are challenging and nonlinear, with a variety of linear and nonlinear inequality constraints.

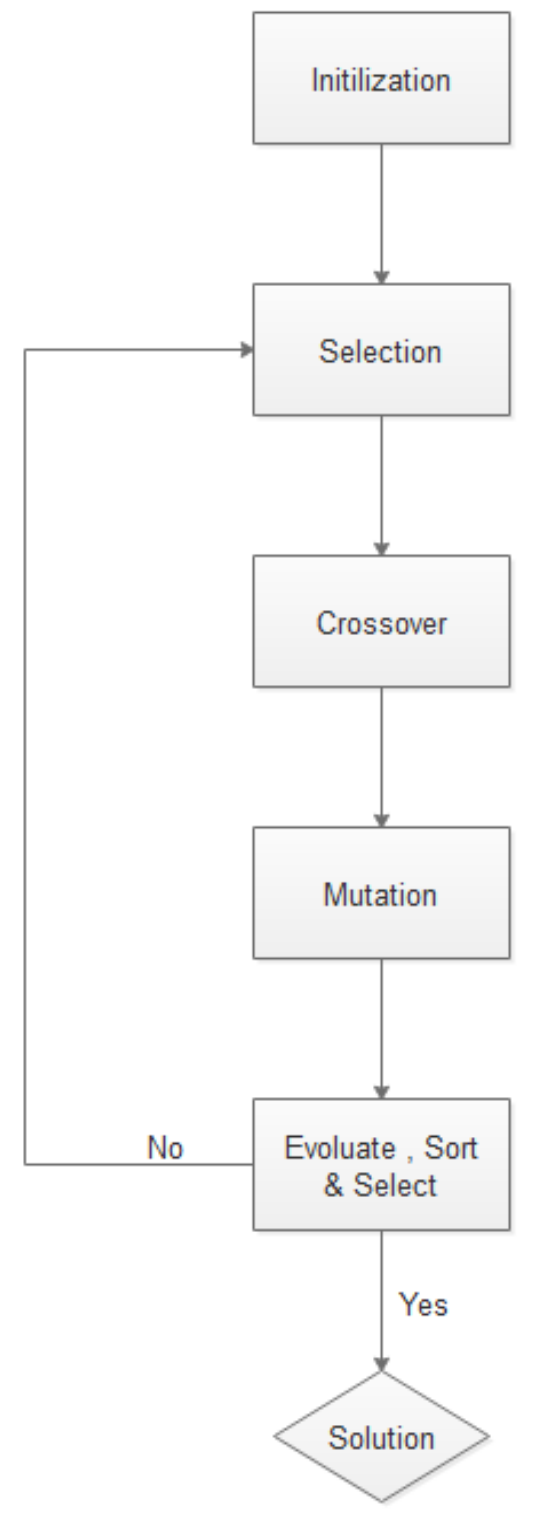

Figure 1: A simple flowchart of GA

\subsection{Himmelblau's non-linear optimization problem}

Himmelblau is the one who came up with the idea for this issue [21]. It has previously been used as a benchmark for numerous evolutionary algorithm-based algorithms [16,20], that need the problem's objective function to be minimized. Five design variables, six nonlinear inequality constraints, and 10 boundary conditions are all present in this problem. 
Five design variables, six nonlinear inequality constraints, and 10 boundary conditions are all present in this problem. The mathematical model of the problem can be described as follows:

$\operatorname{Min} f(x)=5.3578547 x_{3}^{2}+0.8356891 x_{1} x_{5}+$ $37.2932239 x_{1}-40792.141$

Subject to

$$
\begin{gathered}
g_{1}(x)=85.334407+0.0056858 x_{2} x_{5}+ \\
0.00026 x_{1} x_{4}-0.0022053 x_{3} x_{5} \\
g_{2}(x)=80.51249+0.0071317 x_{2} x_{5}+ \\
0.0029955 x_{1} x_{2}-0.0021813 x_{3}^{2} \\
g_{3}(x)=9.300961+0.0047026 x_{3} x_{5}+ \\
0.0012547 x_{1} x_{3}+0.0019085 x_{3} x_{4}
\end{gathered}
$$

Where boundary conditions are,

$$
\begin{aligned}
& 0 \leq g_{1}(x) \leq 92 \\
& 90 \leq g_{2}(x) \leq 110 \\
& 20 \leq g_{3}(x) \leq 25
\end{aligned}
$$

$$
\begin{aligned}
& 78 \leq x_{1} \leq 102 \\
& 33 \leq x_{2} \leq 45 \\
& 27 \leq x_{3}, x_{4}, x_{5} \leq 45
\end{aligned}
$$

\subsection{Pressure Vessel Design}

Pressure vessel is utilized in a variety of industries, including petroleum refining, chemical, power, food and beverage, and pharmaceuticals. Pressure vessel design is considered as one of well-known real-life constrained optimization problems, that has been investigated in a number of recent studies $[16,18,19]$. This issue is about optimizing the design of a cylindrical pressure vessel with hemispherical heads on both sides. Minimizing material cost of each shell, forming, and welding is being an objective investigated in the pressure vessel issue. Where it is considered as a compressed air storage tanks with a working pressure of 2,000 psi and a minimum capacity of $750 \mathrm{ft}^{3}$. The design variables of pressure vessel design are shown in Figure 3. There are four constraints on this problem. The following is how these constraints, and the problem are formulated:

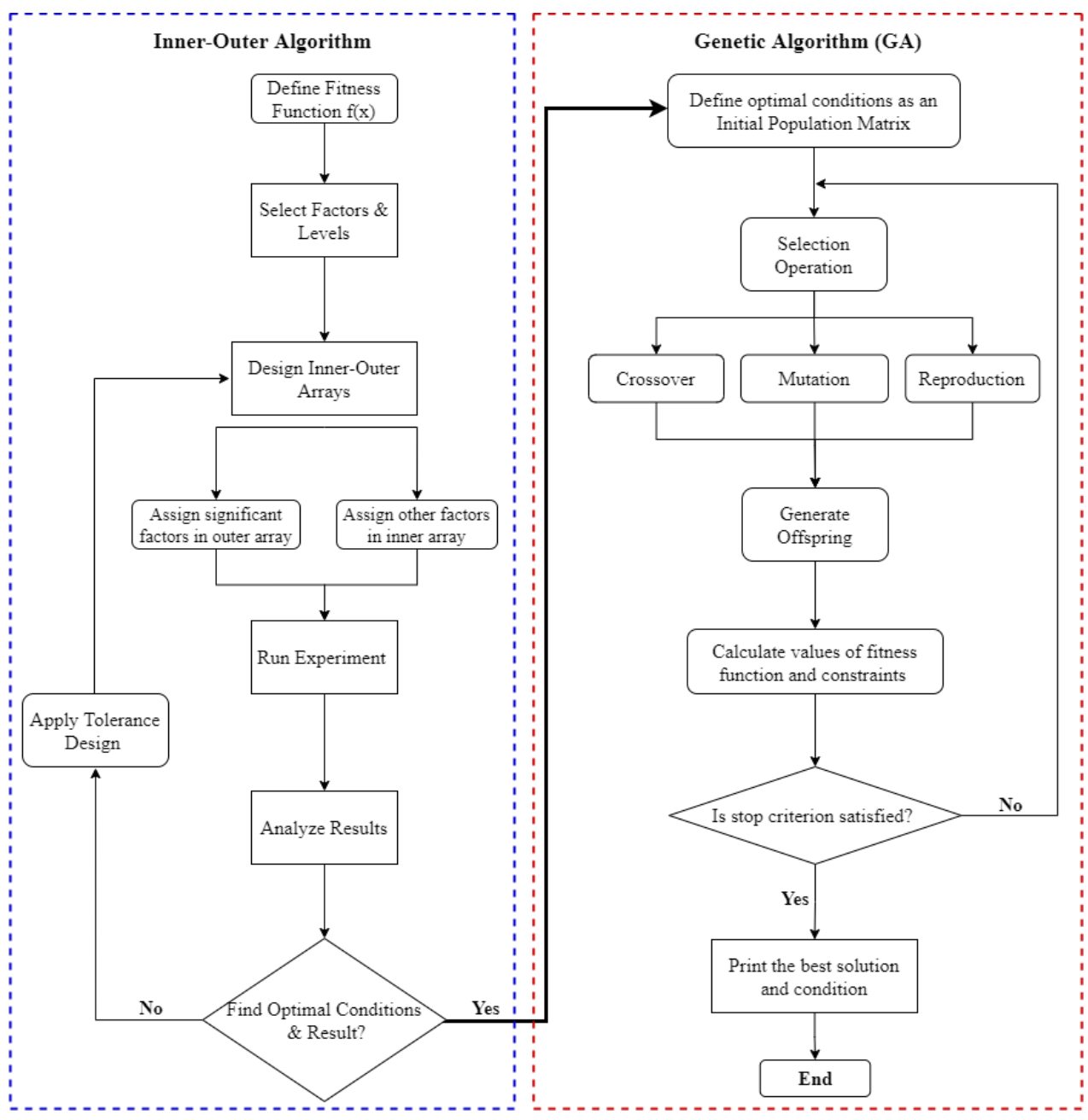

Figure 2: Proposed Model 


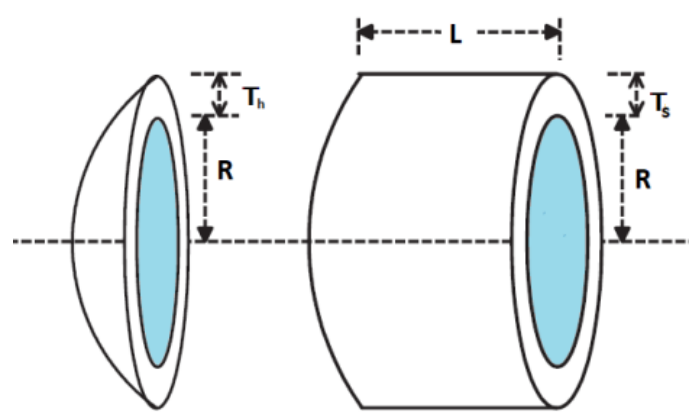

Figure 3 Pressure vessel problem

$x_{1}$ Thickness of the pressure vessel $\left(T_{s}\right)$

$x_{2}$ Thikness of the head $\left(T_{h}\right)$

$x_{3}$ Inner radius of the vessel $(R)$

$x_{4}$ Length of the vessel without heads $(L)$

The issue's objective function $\mathrm{f}(\mathrm{x})$ is to reduce material costs as much as possible. This issue can be mathematically modeled as follows:

$$
\begin{aligned}
& \operatorname{Min} f(x)=0.6224 x_{1} x_{3} x_{4}+1.7781 x_{2} x_{3}^{2}+ \\
& 3.1661 x_{1}^{2} x_{4}+19.84 x_{1}^{2} x_{3}
\end{aligned}
$$

Subject to

$$
\begin{aligned}
& g_{1}(x)=-x_{1}+0.0193 x_{3} \leq 0 \\
& g_{2}(x)=-x_{2}+0.0095 x_{3} \leq 0 \\
& g_{3}(x)=-\pi x_{3}^{2} x_{4}-\frac{4}{3} \pi x_{3}^{3}+1296000 \leq 0 \\
& g_{4}(x)=x_{4}-240 \leq 0
\end{aligned}
$$

Where boundary conditions are,

$$
\begin{aligned}
& 1 \times 0.0625 \leq x_{1}, x_{2} \leq 99 \times 0.0625 \\
& 10 \leq x_{3}, x_{4} \leq 200
\end{aligned}
$$

\section{RESULTS AND DISCUSSION}

Both stages Inner-Outer Arrays and Tolerance Design were kept run on MINITAB 19. Confirmation experiments of 10 simulation runs for pressure vessel and 20 for Himmelblau problem are carried out with the optimum setting along with the default setting parameters of GA MATLAB code. The hybrid algorithm has been run in MATLAB (R2017b) environment on PC outfitted with 64bit OS, Intel Core i5-4590 CPU @ 3.30 GHz, and 8.00 GB RAM.

Both real-life problems mentioned here are obtained in several recent research using different hybrid optimization techniques like Self-Organizing Migrating Genetic Algorithm (SOMGA) [16], Hybrid Genetic AlgorithmParticle Swarm Optimization- Sequential Quadratic Programming (GA-PSO-SQP) [16], Cohort Intelligence algorithm (CI) [18] and Artificial Bee Colony Algorithm (ABC) [19].

Table 1 represent the comparisons between the solutions obtained from SOMGA and that obtained from GA-PSOSQP in terms of the best solution for Himmelblau's nonlinear issue. Then the best one of them is compared with the obtained results from the proposed hybrid algorithm

\begin{tabular}{|c|c|c|c|c|c|}
\hline & \multicolumn{3}{|c|}{ Proposed Algorithms } & \multirow{3}{*}{ Pure GA } & \multirow{3}{*}{$\begin{array}{c}\text { Reported Result } \\
{[16,20]}\end{array}$} \\
\hline & \multirow{2}{*}{ IOA } & Tolerance Design Stage & \multirow{2}{*}{ IOA-GA } & & \\
\hline & & $1 \%$ & & & \\
\hline$x_{1}=$ & 78 & 78 & 78.00139 & 79.03241 & 78 \\
\hline$x_{2}=$ & 33 & 33 & 33.00682 & 36.97643 & 33 \\
\hline$x_{3}=$ & 27 & 27.27 & 27.07399 & 33.4611 & 27.06897 \\
\hline$x_{4}=$ & 45 & 45 & 44.99996 & 36.16495 & 45 \\
\hline
\end{tabular}
IOA-GA, as indicated in Table 2. It is clear that the solution of IOA-GA, which equal -31025.7277 , is better than that obtained from SOMGA, and also the value of design variables satisfied all constraints.

Table 1: Reported Results for Himmelblau's* Nonlinear Problem

\begin{tabular}{|c|c|c|}
\hline Previous Works & Fitness Function $\boldsymbol{f}(\boldsymbol{x})$ & Design Variables \\
\hline $\begin{array}{c}\text { Yassin et al [16] } \\
\text { SOMGA }\end{array}$ & -31025.6689 & $x_{1}=78, \quad \begin{array}{c}x_{2}=33, \\
x_{4}=45, \quad x_{5}=44.967\end{array}$ \\
\hline $\begin{array}{c}\text { Kusum and Singh [20] } \\
\text { GA-PSO-SQP }\end{array}$ & -31025.6 & $x_{3}=27.06897, \quad x_{1}=78, \quad x_{2}=33.0001$, \\
$x_{3}=27.071, \quad x_{4}=45, \quad x_{5}=44.969$ \\
\hline
\end{tabular}

Table 2: Results of Himmelblau's* problem 


\begin{tabular}{|c|c|c|c|c|c|}
\hline $\boldsymbol{x}_{\mathbf{5}}=$ & 45 & 44.55 & 44.95177 & 32.25639 & 44.967 \\
\hline $\boldsymbol{g}_{\mathbf{1}}(\boldsymbol{x})=$ & 91.0117 & 92.00128 & 91.99923 & 90.47853 & 91.99991 \\
\hline $\boldsymbol{g}_{\mathbf{2}}(\boldsymbol{x})=$ & 96.86475 & 97.37436 & 97.20716 & 95.3275 & 97.20741 \\
\hline $\boldsymbol{g}_{\mathbf{3}}(\boldsymbol{x})=$ & 20.48686 & 20.02489 & 19.999 & 20.00043 & 19.99891 \\
\hline $\boldsymbol{f}(\boldsymbol{x})=$ & -29179.5274 & -30994.9481 & -31025.7277 & -29713.3 & -31025.6689 \\
\hline
\end{tabular}

* Himmelblau's problem is an unitless problem [16,20] that was presented before as a benchmark for several evolutionary algorithm-based techniques.

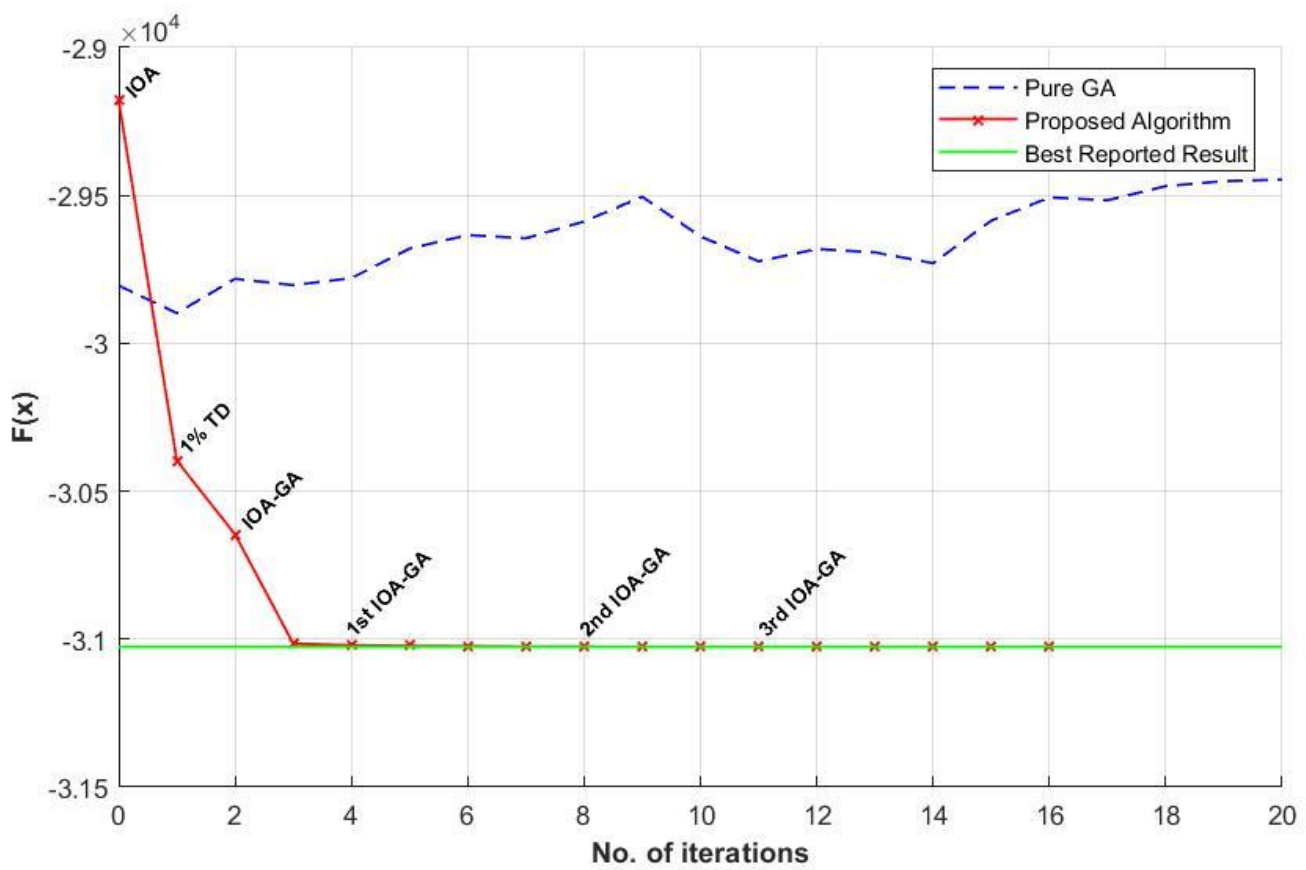

Figure 4: Himmelblau's non-linear function

Figure 4 plots the convergence progress for Himmelblau's nonlinear problem over 20 runs for GA and hybrid IOA-GA comparing with the best reported result at 31025.6689. we graphically show in Fig. 3, the overall evolution of the GA is exhibited by improving individual behaviors using IOA, and this is clear when applying 1st, 2nd, and 3rd Initial Population.
Likewise, in terms of the optimal solution for the pressure vessel case study, Table 3 compares the solutions found from $\mathrm{CI}$ and those acquired via $\mathrm{ABC}$. The best of these is then compared to the results obtained by the suggested hybrid algorithm IOA-GA, as shown in table 4.

The IOA-GA solution is 5912.577, it is obvious that it is superior to the $\mathrm{CI}$ and $\mathrm{ABC}$ solutions. Furthermore, all design constraints are met, unlike the best stated result from $\mathrm{ABC}$ and $\mathrm{CI}$.

Table 3: Reported Results for Pressure Vessel Problem

\begin{tabular}{|c|c|c|c|}
\hline Previous Works & Fitness Function $f(x)$ & \multicolumn{2}{|c|}{ Design Variables } \\
\hline $\begin{array}{c}\text { Yassin et al [16] } \\
\text { SOMGA }\end{array}$ & 6079.974361 & $\begin{array}{r}x_{1}=0.880944 \\
x_{3}=45.634248\end{array}$ & $\begin{aligned} x_{2} & =0.433702 \\
x_{4} & =137.24998\end{aligned}$ \\
\hline $\begin{array}{l}\text { Shastri et al. [18] } \\
\text { ABC }\end{array}$ & 6059.71438 & $\begin{array}{c}x_{1}=0.81249 \\
x_{3}=42.098446\end{array}$ & $\begin{array}{c}x_{2}=0.4375 \\
x_{4}=176.636\end{array}$ \\
\hline $\begin{array}{c}\text { Akay and Karaboga [19] } \\
\text { CI }\end{array}$ & 6059.714339 & $\begin{array}{r}x_{1}=0.8125 \\
x_{3}=42.098446,\end{array}$ & $\begin{array}{l}x_{2}=0.4375 \\
x_{4}=176.63659\end{array}$ \\
\hline
\end{tabular}


The solution convergence graph for pressure vessel design function is presented in Figure 5and compared with results of pure GA and reported result from CI algorithm. The general development of Pure GA is displayed by enhancing individuals utilizing IOA. This is obvious after employing the 1st, 2nd, and 3rd Initial Populations for hybrid IOA-GA algorithm, as shown in the partial zooming graph on the right side of Figure 5. The chromosomes value used during hybrid IOA-GA are illustrated in Table 5.

Table 4: Results of Pressure Vessel* Design

\begin{tabular}{|c|c|c|c|c|c|c|c|}
\hline & \multicolumn{5}{|c|}{ Proposed Algorithms } & \multirow{3}{*}{$\begin{array}{l}\text { Pure } \\
\text { GA }\end{array}$} & \multirow{3}{*}{$\begin{array}{c}\text { Reported } \\
\text { Result } \\
{[14]}\end{array}$} \\
\hline & \multirow{2}{*}{ IOA } & \multicolumn{3}{|c|}{ Tolerance Design Stage } & \multirow{2}{*}{$\begin{array}{l}\text { IOA- } \\
\text { GA }\end{array}$} & & \\
\hline & & $5 \%$ & $0.5 \%$ & $0.1 \%$ & & & \\
\hline$x_{1}=$ & 2.104167 & 1.998959 & 1.799063 & 1.43925 & 0.793788 & 1.538971 & 0.81249 \\
\hline$x_{2}=$ & 1.59375 & 1.514062 & 1.362656 & 1.090125 & 0.39237 & 0.763017 & 0.4375 \\
\hline$x_{3}=$ & 57.5 & 54.625 & 49.1625 & 39.33 & 41.12887 & 79.72609 & 42.09844 \\
\hline$x_{4}=$ & 73.3333 & 69.66664 & 62.69998 & 50.15998 & 189.0334 & 186.5357 & 176.636 \\
\hline$g_{1}(x)=$ & -0.994417 & -0.9446965 & -0.85022675 & -0.680181 & $-5.4 \mathrm{E}-07$ & -0.00026 & $9.89 \mathrm{E}-06$ \\
\hline$g_{2}(x)=$ & -1.0452 & -0.9929395 & -0.89364575 & -0.7149168 & -0.38283 & -0.75348 & -0.42796 \\
\hline$g_{3}(x)=$ & -262033.2604 & -39818.81351 & 322188.0546 & 797408.3034 & -0.54969 & -8261657 & $3.703597 * *$ \\
\hline$g_{4}(x)=$ & -166.6667 & -170.33336 & -177.30002 & -189.84002 & -50.9666 & -53.4643 & -63.364 \\
\hline$f(x)=$ & 20970.58901 & 17979.65916 & 13107.1720 & 6710.87089 & $\underline{5912.577}$ & 47464.69 & 6059.630523 \\
\hline
\end{tabular}

* Pressure vessel design problem was presented in several works $[16,18,19]$ and the unit of variables is inch.

** This constraint was not satisfied.

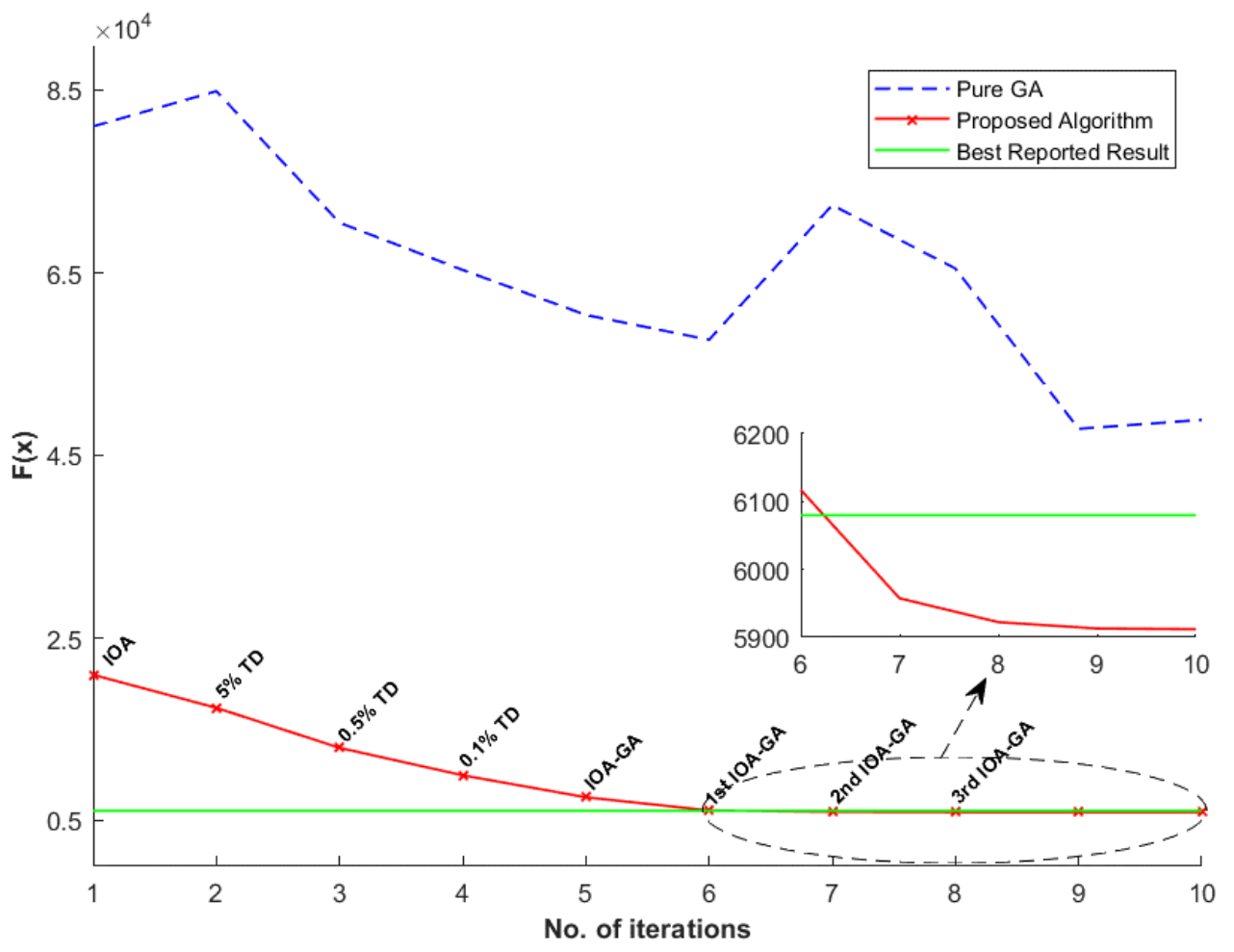

Figure 5: Pressure vessel function 
Table 5: Initial Populations for IOA-GA

\begin{tabular}{|c|c|c|c|c|}
\hline \multirow{2}{*}{ Initial Population } & \multicolumn{4}{|c|}{ Chromosomes } \\
\cline { 2 - 5 } & $\boldsymbol{x}_{\mathbf{1}}$ & $\boldsymbol{x}_{\mathbf{2}}$ & $\boldsymbol{x}_{\mathbf{3}}$ & $\boldsymbol{x}_{\mathbf{4}}$ \\
\hline 1st & 0.80650439 & 0.442142539 & 41.78682636 & 181.281381 \\
\hline 2nd & 0.806666202 & 0.398736325 & 41.79614733 & 180.4190671 \\
\hline 3rd & 0.794685191 & 0.392812549 & 41.17482025 & 188.4310888 \\
\hline
\end{tabular}

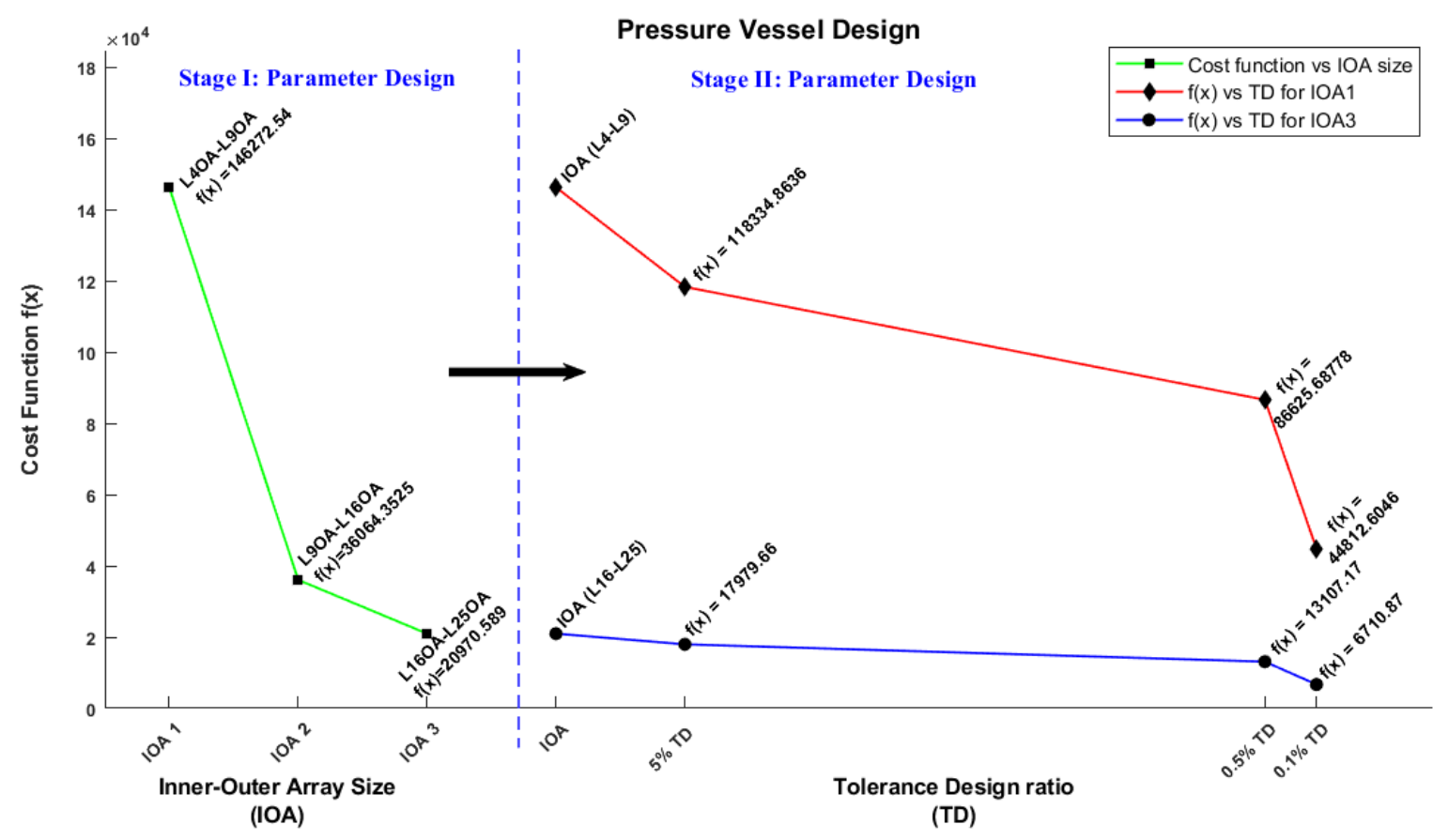

Figure 6 IOA stages for solving pressure vessel design problem

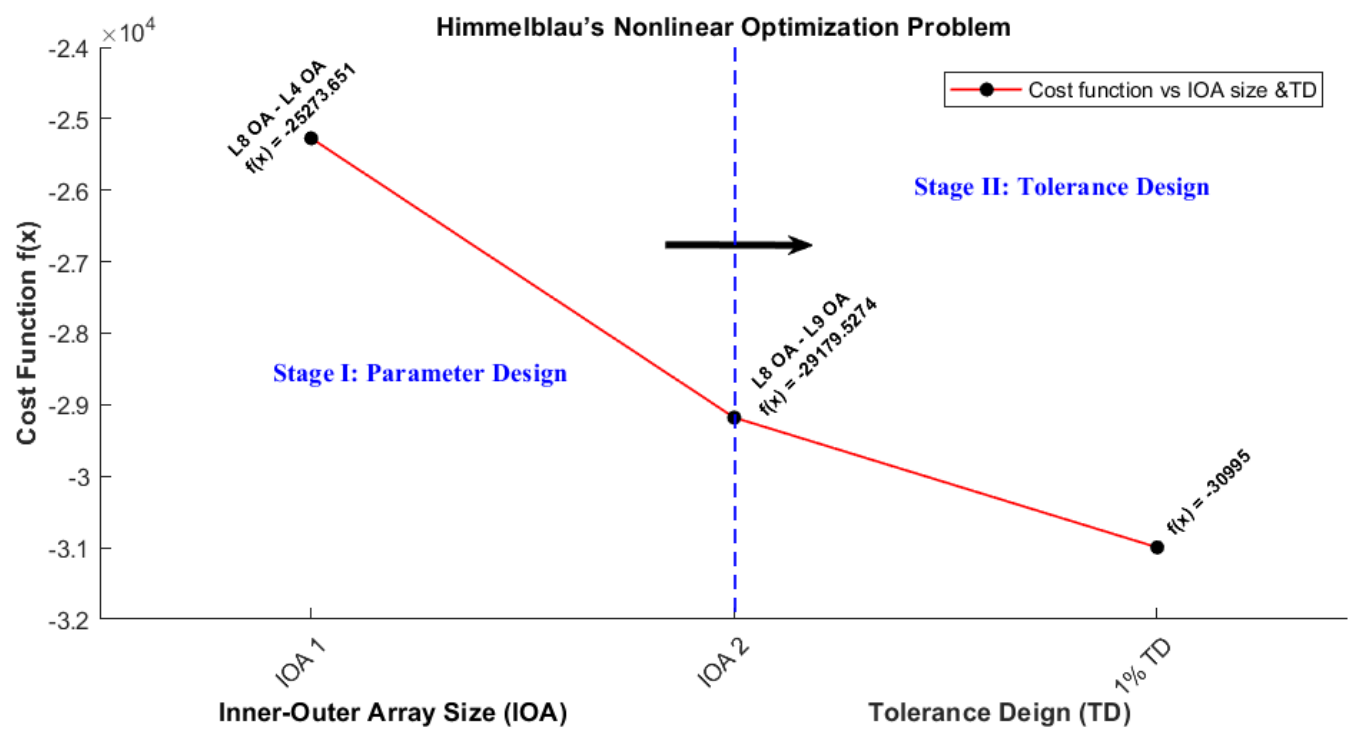

Figure 7: IOA stages for solving Himmelblau's Nonlinear Optimization Problem 
The stages of the Inner-Outer procedure are shown in detail in Figure 6 and 7, which are as follows:

- Stag I: Parameter Design

- Stage II: Tolerance Design.

Figure 6 illustrates the first stage of parameter design, which included selecting appropriate arrays to describe the pressure vessel problem. In this stage, (L4-L9) IOA, (L9L16) IOA and (L16-L25) IOA are designed to study the effect of IOA size on cost function $\mathrm{f}(\mathrm{x})$, in order to select the most appropriate IOA for the nature of this problem, and then proceed to the second stage, Tolerance design. The best result from the previous stage is analyzed in the second stage, where the levels surrounding that result are explored in a tight range. In pressure vessel problem, (L4-L9) IOA and (L16-L25) IOA have progressed to the tolerance design stage, where their findings will be investigated at various tolerance design ratios, including: $5 \%, 0.5 \%$, and $0.1 \%$. Thus, after designing the tolerance for their levels, it is possible to determine whether their behavior is close to the global optimal solution or not. It is obvious that utilizing the (L16-L25) IOA and then the design tolerance ratios produced the greatest result in terms of being near to the global optimum value.

Likewise, in Himmelblau's non-linear optimization problem, (L8-L4) IOA and (L8-L9) IOA are created to investigate the effect of IOA size on the cost function $f(x)$, as shown in Figure 7, afterwards choose the most appropriate IOA for this problem, and then move on to Stage II. The result of (L8-L9) IOA was the best one, so its outcome levels were analyzed using a tolerance design ratio $1 \%$. The result is satisfied, which is near to the global result found in earlier studies.

\section{CONCLUSION}

The major challenge facing optimization is that any standalone optimizer is unable to obtain the global optimal value, particularly when the problems are complex, and lager sized. Therefore, this paper presents an efficient approach aiding in overcoming premature convergence to local optimum or no convergence at all., called Inner-Outer Array (IOA). The IOA is developed to enhance optimizer's performance in finding the most feasible region with the optimal solution. This strategy helps to avoid going more deeper into the optimizer's parameter settings, which are not appropriate in all cases. Consequently, a novel hybridization approach combining Inner-Outer Array (IOA) and Genetic Algorithm (GA), called Hybrid IOA-GA, was proposed in this work. The IOA feeds the genetic algorithm with an initial population to guide it get the global or near global optimum solution as fast as possible. To validate the performance of the proposed approach IOA-GA, approximately 15 real-life mechanical engineering optimization problems were used. In this article, Himmelblau's non-linear optimization problem and pressure vessel design problem utilized as case studies to demonstrate the approach's robustness. Thereafter, the findings were compared to more complicated and wellknown algorithms like SOMGA, GA-PSO-SQP, CI, and
ABC. In Himmelblau's nonlinear optimization problem, the difference proportion between results proposed Hybrid IOA-GA and SOMGA is equal $\mathbf{0 . 0 0 0 1 8 9 \%}$. In pressure vessel design problem, the difference proportion between results proposed IOA-GA and $\mathrm{ABC}$, or $\mathrm{CI}$, is equal $\mathbf{- 2 . 4 2 6 \%}$ (negative means that IOA-GA result is better than that reported). The result indicated that the suggested method is capable when compared to existing hybridization procedures. Ultimately, the IOA-GA method performs well in comparison to other effective methods.

\section{Credit Authorship Contribution Statement:}

Omnia O. FAdel: Carrying out the methodology, developing computational technique \& validation, Original draft preparation. Mohamed H. Gadallah: Generating the idea, Validation, Editing, Reviewing \& Supervision, Original draft preparation. Mohamed A. Zaghloul: Reviewing and Editing. Hanan K. Kouta: Reviewing and Editing.

\section{Declaration of competing Interest}

The authors declare that they have no known competing financial interests or personal relationships that could have appeared to influence the work reported in this paper.

\section{References}

[1] G. D. Angelo, and F. Palmieri. GGA: A modified genetic algorithm with gradient-based local search for solving constrained optimization problems. Information Sciences, 547, 202, 136-162.

[2] O.O. Fadel, M.H. Gadallah, H.K. Kouta and M.A. Zaghloul. Hybrid Inner-Outer Algorithm for Solving Real-World Mechanical Optimization Problems. Journal of Engineering and Applied Science 68, no. 1, 2021, pp. $1-18$.

[3] J.T. Tsai, T.K. Liu and J.H. Chou. Hybrid Taguchigenetic algorithm for global numerical optimization. IEEE Transactions on evolutionary computation, 2004, 8(4): pp. 365-377.

[4] P.A. Vaghela and J.M. Prajapati. Hybridization of Taguchi and genetic algorithm to minimize iteration for optimization of solution. MethodsX 6, 2019, pp. 230238.

[5] I. Karen, A.R. Yildiz, N. Kaya, Öztürk NURSEL, and Oeztuerk Ferruh. Hybrid approach for genetic algorithm and Taguchi's method-based design optimization in the automotive industry. Int J Prod Res 44(22), 2006, pp. 4897-4914.

[6] S. Picek, M. Golub and D. Jakobovic. Influence of the crossover operator in the performance of the hybrid Taguchi GA. In: 2012 IEEE Congress on Evolutionary Computation, 2012, pp. 1-8.

[7] H.S. Bernardino, H.J. Barbosa, and A.C. Lemonge, A Hybrid Genetic Algorithm for Constrained Optimization Problems in Mechanical Engineering. IEEE, 2007.

[8] F.T. Chan, S.H. Chung, and Wadhwa. A Hybrid Genetic Algorithm for Production and Distribution. The 
International Journal of Management Science, Elsevier, 2004.

[9] D.J. Mala, E. Ruby, and V. Mohan. A Hybrid Test Optimization Framework Coupling Genetic Algorithm with Local Search Technique. Computing and Informatics, Vol. 29, 2010.

[10] K.S. Sangwan, S. Saxena, and G. Kan. Optimization of Machining Parameters to Minimize Surface Roughness Using Integrated ANN-GA Approach. CIRP, Elsevier, 2015.

[11] G. Kant, and K.S. Sangwan. Predictive Modeling and Optimization of Machining Parameters to Minimize Surface Roughness using ANN Coupled with GA. CIRP, Elsevier, 2015.

[12] A.F. Ali, and M.A. Tawhid. A Hybrid Particle Swarm Optimization and Genetic Algorithm with Population Partitioning for Large Scale Optimization Problems. Ain Shams Engineering Journal, Production and hosting by Elsevier, 2016.

[13] W. Javaid, A. Tariq, and I. Hussain. A Comparison of a Standard Genetic Algorithm with a Hybrid Genetic Algorithm Applied to Cell Formation Problem. Advances in Mechanical Engineering, 2014.

[14] N. Al-Milli. Hybrid Genetic Algorithm with Great Deluge to Solve Constrained Optimization Problems. Journal of Theoretical and Applied Information Technology, 2014.

[15] T. Zhang, Y. Liu, Y. Rao, X. Li, and Q. Zhao. Optimal design of building environment with hybrid genetic algorithm, artificial neural network, multivariate regression analysis and fuzzy logic controller. Building and Environment, 175, 2020, pp. 106810.

[16] Y. Belkourchia, L. Azrar, and E.S.M. Zeriab. A Hybrid Optimization Algorithm for Solving Constrained Engineering Design Problems. IEEE, 2019.

[17] A. Duary, M.S. Rahman, A.A. Shaikh, S.T. Niaki, and AK. Bhunia. A new hybrid algorithm to solve boundconstrained nonlinear optimization problems. Neural Computing and Applications, Jan 2020.

[18] A.S. Shastri, E.V. Thorat, A.J. Kulkarni, and P.S. Jadhav. Optimization of constrained engineering design problems using cohort intelligence method. In Proceedings of the 2nd International Conference on Data Engineering and Communication Technology, Springer, Singapore, 2019.

[19] B. Akay and D. Karaboga. Artificial bee colony algorithm for large-scale problems and engineering design optimization. Journal of Intelligent Manufacturing, 23(4), 2012, pp.1001-1014.

[20] K. Deep and D. Singh. Engineering Optimization Using SOMGA. In Proceedings of the Second International Conference on Soft Computing for Problem Solving, Springer, New Delhi, 2014.

[21] D.M. Himmelblau. Applied Nonlinear Programming. McGraw-Hill, New York, 1972. 